\title{
Pre-operative Embolization of an Intraventricular Meningioma Using Onyx
}

\author{
Andrew S Jack, Jian-Qiang Lu, Robert A Ashforth, Robert W Broad, Tim E Darsaut
}

Keywords: Intraventricular meningioma, Intraventricular tumour, Onyx, Pre-operative embolization

Intraventricular meningiomas are primarily a surgical disease. One of the most important aspects in their operative treatment is controlling their arterial blood supply. ${ }^{1}$ In an attempt to reduce the vascularity of meningiomas, pre-operative particulate embolization is often used. However, the potential for unwanted reflux within the intracranial vasculature usually limits this approach to those lesions supplied by arteries from the external circulation. Because intraventricular meningiomas are supplied by branches from the intracranial circulation, these lesions are not typically embolized due to the risk of particulate reflux.

Onyx (Covidien, Irvine, CA, USA) is a liquid embolic agent that potentially offers better operator control than particles and may be appropriate for embolization of some tumours. Here we present a case of an intraventricular meningioma with arterial supply from a lateral posterior choroidal artery in which Onyx was used to successfully devascularize the lesion prior to operation.

\section{Case Report}

A 22-year old previously healthy woman presented with a two-month history of progressive headache, nausea, and vomiting. Examination revealed decreased sensation to the left side of her face and arm and a subtle left-sided pronator drift. Cranial computed tomography (CT ) (Figure 1A) followed by magnetic resonance imaging (MRI) (Figure 1B) revealed a large homogeneously enhancing tumour within the right atrium of the lateral ventricle with imaging characteristics consistent with a meningioma.

Cerebral angiography was performed to delineate the vascular anatomy of the tumour for surgical planning. This revealed hypertrophied medial and lateral posterior choroidal arteries supplying the tumour (Figure 2A and B), with substantial tumour blush. In an effort to decrease tumour vascularity, the patient underwent embolization with Onyx 18. Under general anaesthetic, an Apollo $1.5 \mathrm{~cm}$ detachable tip microcatheter (Covidien, Irvine, CA, USA) was navigated into the hypertrophic lateral posterior choroidal vessel (Figure $2 \mathrm{~B}$ and $2 \mathrm{C}$ ). After flushing with dimethyl sulfoxide (DMSO), the tumour was successfully devascularized with Onyx, which took less than 10 seconds due to rapid reflux, and required less than one $\mathrm{mL}$ of liquid embolic (Figure 2D-F). The microcatheter tip detached readily and easily. The endovascular procedure was well tolerated, and she was scheduled for open tumour resection the following day.

Via temporal craniotomy, a corticectomy was made in the middle temporal gyrus. The exposure was carried down into the ventricle where the tumour capsule was identified. Greyish-tan in color, the capsule was coagulated and the tumour debulked internally with the Cavitron ultrasonic aspirator (CUSA, Integra Radionics, Plainsboro, NJ, USA). The capsule was mildly adherent to the choroid plexus but the tumour was completely resected with what was judged by the surgeon to be minimal blood loss.

The pathology examination revealed an atypical, World Health Organization grade II meningioma (Figure 3). The meningioma exhibited moderate hypercellularity and pleomorphism, immunoreactivity for epithelial membrane antigen (Figure 3A), and intratumoural vessels were filled with the Onyx embolizate (Figure 3B). Mitoses were readily found (Figure 3C), as many as six per ten high power fields. The MIB-1 labelling index of proliferation was as high as five percent. In addition to the Onyx embolizate, there were abundant infiltrating foamy and CD68-immunoreactive macrophages (Figure 3C and 3D).

The patient had an uncomplicated post-operative course and was discharged home on post-operative day five. At no point did she require a blood transfusion. At six-month follow-up, she remained asymptomatic and neurologically intact. Post-operative $\mathrm{CT}$ and contrast enhanced MRI (Figure 1C and 1D) revealed complete resection of the tumour with no evidence of recurrence.

\section{DiscuSSION}

Pre-operative embolization of meningiomas was first described in the early 1970s. ${ }^{2}$ Proponents of this surgical adjunct cite decreased operative blood loss, increased tumour necrosis, and decreased operative time as reasons for devascularization preoperatively. ${ }^{2}$ However, risks such as stroke or puncture-related complications typically limit the use of this adjunct to those cases where benefits are thought to outweigh risks. ${ }^{2}$

Onyx is an ethylene vinyl alcohol copolymer DMSO solvent. Tantalum is also added to increase radio-opacity. Upon contact with aqueous solutions, including blood, the solvent dissipates and the liquid Onyx forms a cast which progressively hardens. This then enables slower and potentially more controlled injections. Typically used to treat arteriovenous malformations

From the Division of Neurosurgery (ASJ,RWB,TED), Department of Surgery;

Neuropathology Section (JL), Department of Laboratory Medicine and Pathology; Department of Radiology and Diagnostic Imaging (RAA), University of Alberta

Hospital, Edmonton, Alberta, Canada

Received December 17, 2014. Final Revisions Submitted March 17, 2015.

Correspondence to: Tim E Darsaut, Division of Neurosurgery, 2D1 WCM, University of Alberta, 8440112 St, Edmonton, Alberta, Canada T6G 2B7. Email: tdarsaut@ualberta.ca 

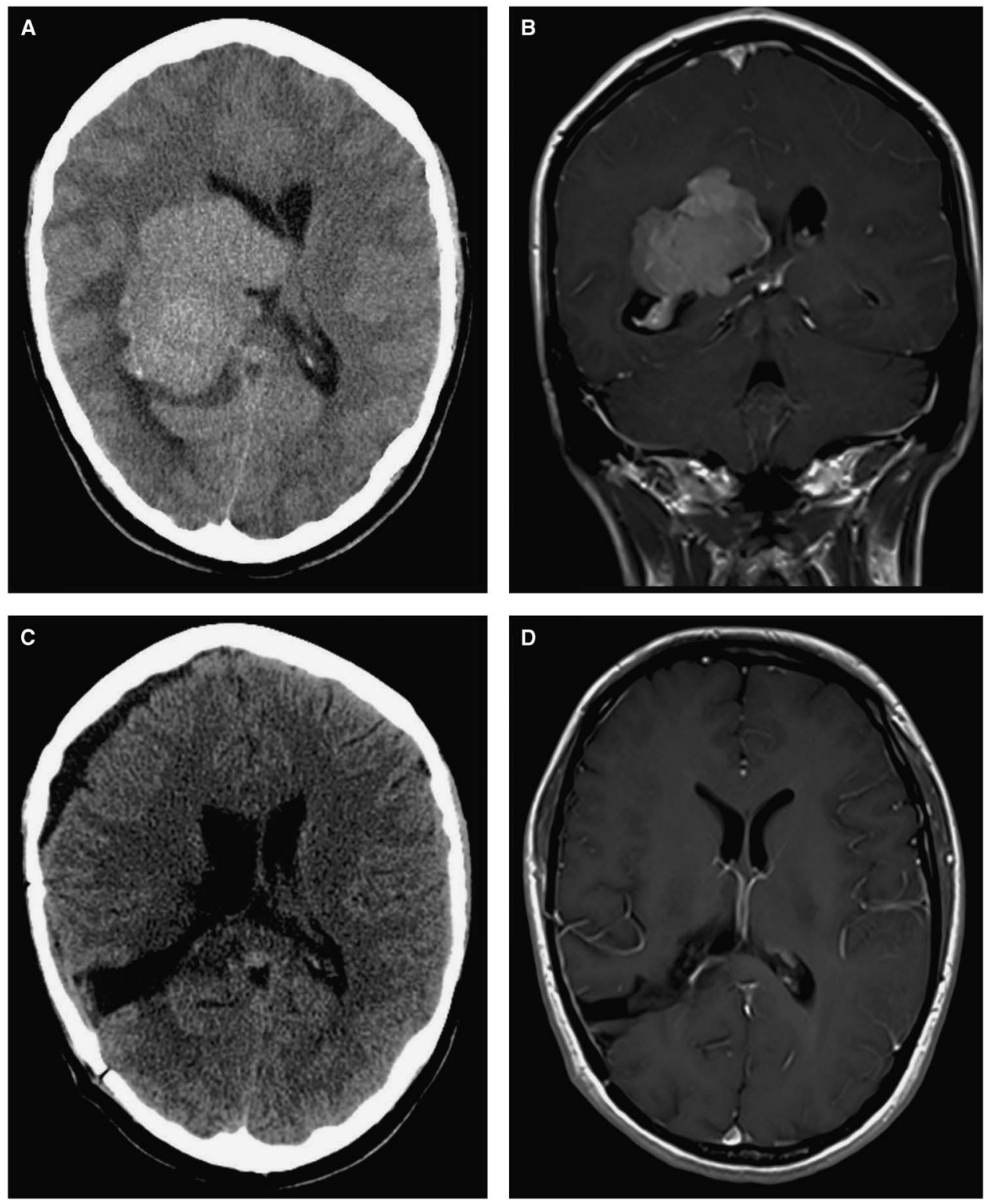

Figure 1: A) Pre-operative CT showing a large $(6.6 \times 3.2 \times 5.2 \mathrm{~cm})$, right atrial intraventricular lesion with significant mass effect; $B)$ pre-operative MRI demonstrating the homogeneously enhancing tumour; C) immediate post-operative CT, and D) six month post-operative MRI showing complete tumour resection.

and dural arteriovenous fistulae, Onyx has also been used to treat some intracranial tumours. ${ }^{3}$ However, this is the first reported use for an intraventricular meningioma.

Previous studies investigating the use of Onyx as an embolizate have suggested decreased operative blood loss and transfusion requirements, as well as decreased operative time and length of hospitalization. ${ }^{1,3,4}$ Although the study failed to show a difference in operative time, degree of resection, or transfusion requirements between the two groups, authors claimed a statistically significant difference in total length of hospitalization. ${ }^{4}$ Others have also reported decreased operative blood loss and transfusion requirements, compared to matched historical controls. $^{5}$
Intraventricular meningiomas are relatively rare, which may account for this lacune in the embolization literature. Their relatively deep location and blood supply renders them favourable for Onyx embolization as particulate embolization carries a high risk of occluding intracranial arteries. Here, because the hypertrophied choroidal vessels enabled catheterization, we were able to deliver Onyx in a controlled fashion and successfully devascularize the tumour. However, this technique still entails considerable risk of stroke which, in this case, could have potentially affected the posterior choroidal arterial distribution (visual field loss), or infarction in the territory supplied by the posterior choroidal arteries, which can also cause visual field or hemisensory loss, and neuropsychological problems. 

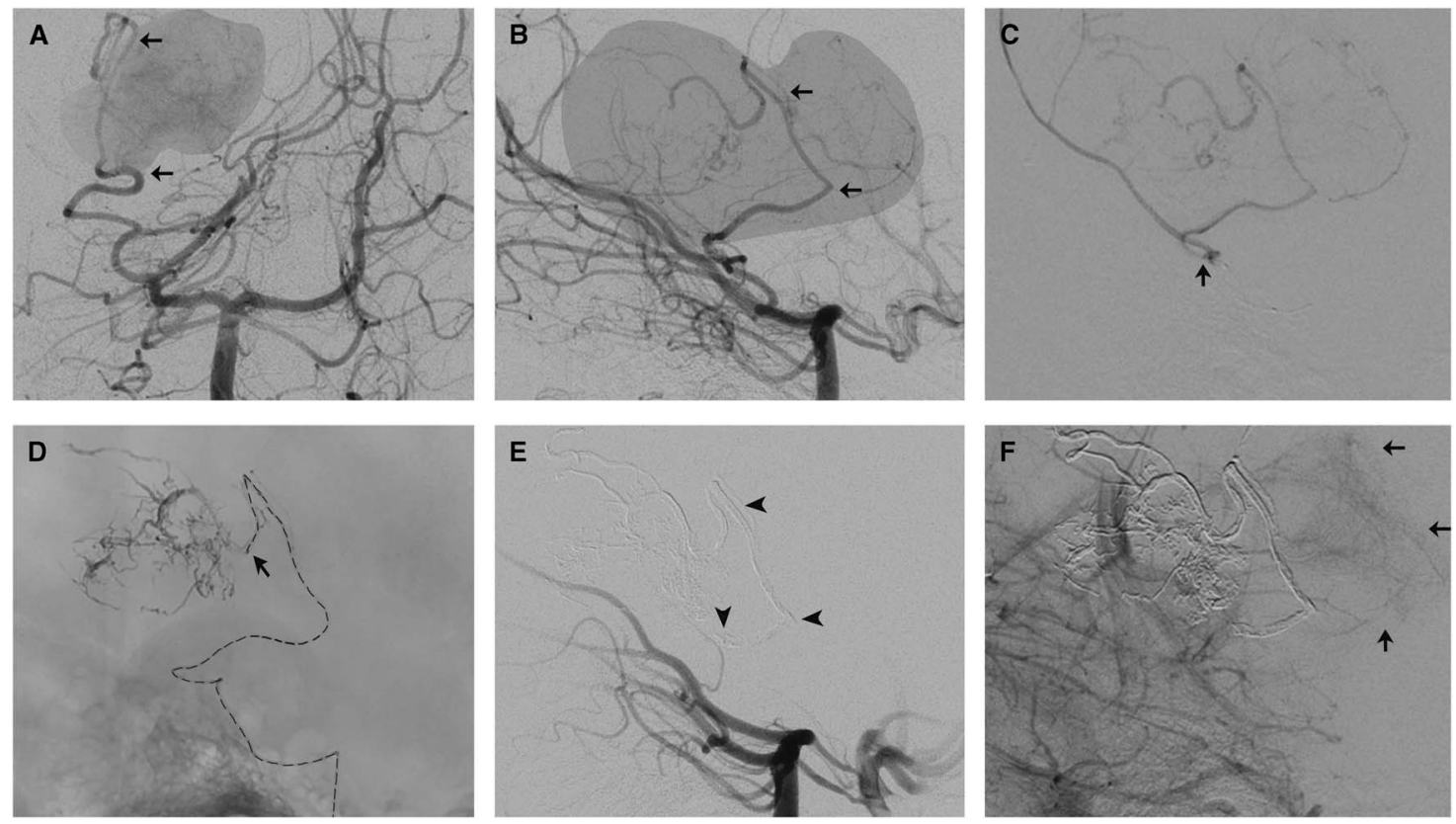

Figure 2: A) Left anterior oblique and B) lateral projection of a left vertebral artery injection demonstrating a prominent right lateral posterior choroidal artery (arrows) supplying the tumour (shaded); C) microcatheter injection showing absence of branches supplying brain, and point of judged maximal permissible reflux (arrow); D) position of the microcatheter tip (arrow) during injection. E+F) Onyx cast showing extent of reflux (arrowheads) and residual tumor blush after embolization (arrows).
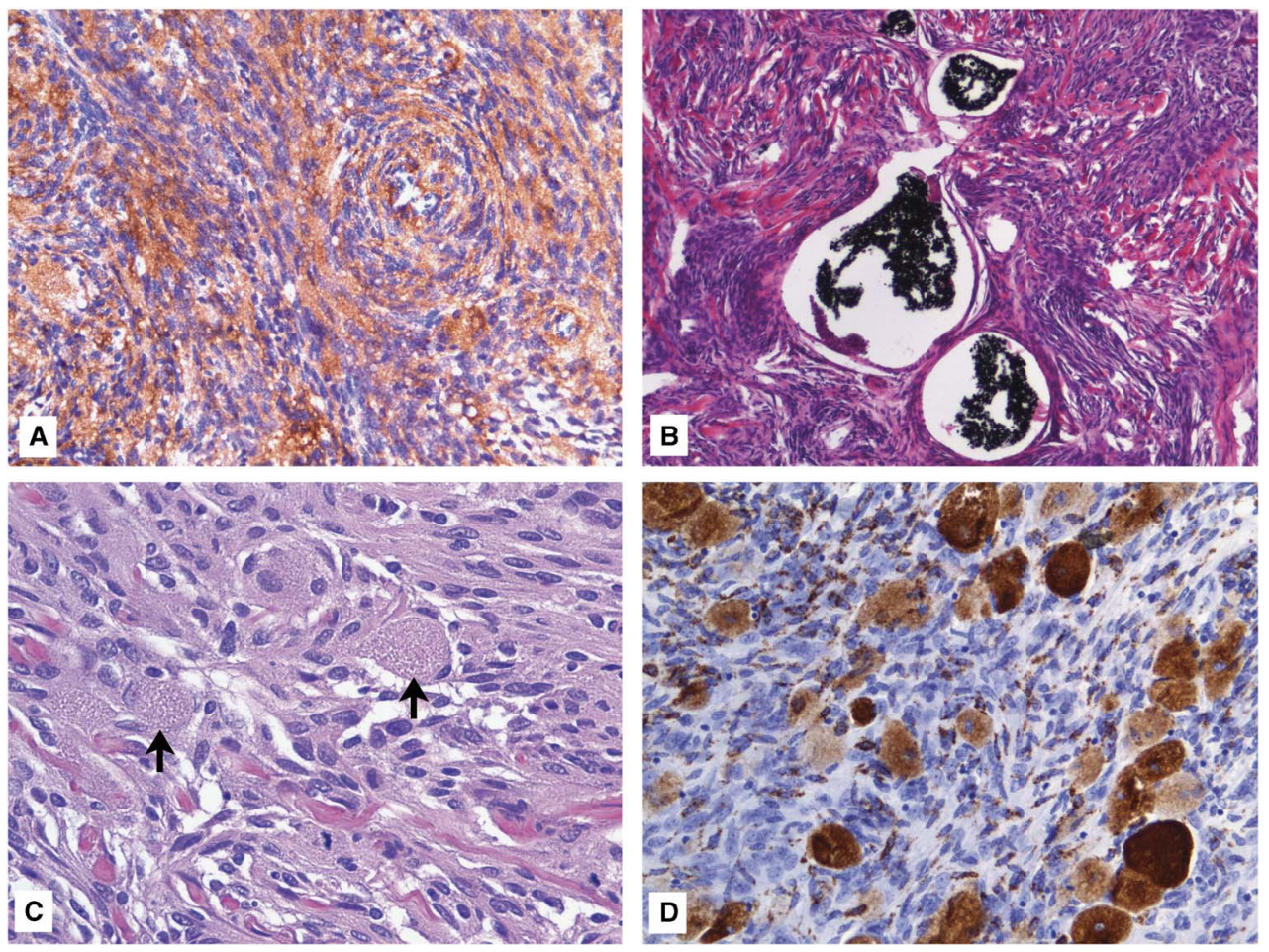

Figure 3: A) Photomicrograph of an intraventricular atypical meningioma showing focal whorl formation and immunoreactivity for epithelial membrane antigen (original magnification $\times 200$ ); B) intratumoural vessels filled with Onyx 18 (original magnification $\times 100$ ); C) the tumour exhibiting frequent foamy macrophages in reaction to the Onyx (arrowheads, original magnification $\times 400$ ); D) CD68 marker highlighting frequent macrophage infiltrates in the tumour (original magnification $\times 200$ ). 


\section{CONCLUSION}

Embolization with a liquid embolic such as Onyx may provide devascularization of intraventricular meningiomas, lesions that would not otherwise be amenable to particulate embolization. This presurgical adjunct still entails considerable risk and careful consideration of the risk versus benefit profile for each patient must be evaluated.

\section{DisClOSURES}

The authors do not have anything to disclose.

\section{REFERENCES}

1. Hirohata $M$, Abe T, Morimitsu H, Fujimura N, Shigemori M, Norbash AM. Preoperative selective internal carotid artery dural branch embolisation for petroclival meningiomas. Neuroradiology. 2003;45:656-60.

2. Shah AH, Patel N, Raper DM, et al. The role of preoperative embolization for intracranial meningiomas. J Neurosurgery. 2013;119:364-72.

3. Gobin YP, Murayama Y, Milanese K, et al. Head and neck hypervascular lesions: embolization with ethylene vinyl alcohol copolymer-laboratory evaluation in Swine and clinical evaluation in humans. Radiology. 2001;221:309-17.

4. Gore P, Theodore N, Brasiliense L, et al. The utility of onyx for preoperative embolization of cranial and spinal tumors. Neurosurgery. 2008;62:1204-11; discussion 11-2.

5. Dean BL, Flom RA, Wallace RC, et al. Efficacy of endovascular treatment of meningiomas: evaluation with matched samples. AJNR Am J Neuroradiology. 1994;15:1675-80. 